Pacific Journal of Mathematics

FEE PRODUCTS AND ELEMENTARY EQUIVALENCE 


\title{
FREE PRODUCTS AND ELEMENTARY EQUIVALENCE
}

\section{PHILIP OLIN}

\begin{abstract}
It is shown that the free product operation on two groupoids preserves both elementary equivalence and elementary subsystem. An example is given showing the above results for semigroups false, thus answering in the negative a question of Feferman and Vaught.
\end{abstract}

In their important paper [3], Feferman and Vaught show, as a consequence of a stronger result, that many of the usual product operations preserve elementary equivalence. For example, if $\mathfrak{A}_{1}, \mathfrak{A}_{2}$, $\mathfrak{B}_{1}, \mathfrak{B}_{2}$ are structures such that $\mathfrak{N}_{1}$ and $\mathfrak{B}_{1}$ have the same elementary first order properties (denoted $\mathfrak{A}_{1} \equiv \mathfrak{B}_{1}$ ) and similarly $\mathfrak{A}_{2} \equiv \mathfrak{B}_{2}$, then for the direct products we have $\mathfrak{A}_{1} \times \mathfrak{A}_{2} \equiv \mathfrak{B}_{1} \times \mathfrak{B}_{2}$. In the footnote on page 76 of that paper they state that their methods do not apply to free products or tensor products, and they ask if these two operations preserve elementary equivalence. The answer is known to be negative for tensor products (see [2], [4]). We show here that for free products the answer is also negative for both elementary equivalence and elementary subsystem. In our counterexample $\mathfrak{A}_{1}, \mathfrak{A}_{2}$, $\mathfrak{B}_{1}, \mathfrak{B}_{2}$ are semigroups, and the idea used is similar to the idea in Example 1.3 of [5].

In that same footnote, Feferman and Vaught mention a method due to Fraïssé and later developed by Ehrenfeucht [1], and they ask if this method might by applied to the problem of preserving elementary equivalence. We show here, using this method of games of Fraïsse-Ehrenfeucht, that the free product operation on groupoids preserves both elementary equivalence and elementary subsystem. A groupoid is simply a nonempty set with a binary function.

Also explicitly mentioned in that footnote is the question whether free products of groups preserve elementary equivalence. We have been unable to answer this question.

It should be noted that the definition of free product depends on the class of structures considered, so that if $\mathfrak{A}$ and $\mathfrak{B}$ are semigroups then their free product as semigroups is different from the free product formed with them by thinking of them as groupoids. At the end of the paper we attempt in a short space to give some motivation for the example and the proof.

The results of this paper were announced in [6].

We denote the sentence $\varphi$ being true in the model $\mathfrak{A}$ by $\mathfrak{A} \vDash \varphi$. If for every elementary first order sentence $\varphi$ we have $\mathfrak{A} \vDash \phi$ iff $\mathfrak{B} \vDash \varnothing$ then $\mathfrak{A}$ and $\mathfrak{B}$ are said to be elementarily equivalent and 
we write $\mathfrak{A} \equiv \mathfrak{B}$. If $\mathfrak{A}$ is a submodel of $\mathfrak{B}$ and if for every $\operatorname{such} \varphi$ with possibly constants from $\mathfrak{A}$ we have $\mathfrak{A} \vDash \varnothing$ iff $\mathfrak{B} \vDash \varnothing$ then we say $\mathfrak{A}$ is an elementary subsystem of $\mathfrak{B}$ and we write $\mathfrak{A} \prec \mathfrak{B}$. The free product operation (over a given, understood class) is denoted by *. For the result for groupoids we assume some familiarity with the method of games (see [1] or [2]).

ExAMPLE. We consider the class of semigroups and now construct semigroups $\mathfrak{A}_{1}, \mathfrak{A}_{2}, \mathfrak{B}_{1}, \mathfrak{B}_{2}$ such that $\mathfrak{A}_{1} \equiv \mathfrak{B}_{1}, \mathfrak{A}_{2} \equiv \mathfrak{B}_{2}$ and yet $\mathfrak{A}_{1} * \mathfrak{A}_{2} \neq \mathfrak{B}_{1} * \mathfrak{B}_{2}$. In fact we will have $\mathfrak{A}_{1} \cong \mathfrak{B}_{1}$, the trivial semigroup; and $\mathfrak{A}_{2} \prec \mathfrak{B}_{2}$, both being denumerably infinite.

Let $\mathfrak{B}$ be the semigroup $\langle B, \cdot\rangle$ where $B$ is the one-element set $\{b\}$ and $b \cdot b=b$. Of course $\mathfrak{B} \prec \mathfrak{B}$. We will now define a semigroup $\mathfrak{A}=\langle A, \cdot\rangle$. The generators of $\mathfrak{A}$ will be the members (all distinct) of the set $G=\left\{a_{i}\right\}_{i<\omega} \cup\left\{c_{j}^{i}\right\}_{i, j<\omega}$. For each $i<\omega$ let $\left\{S_{j}^{i}\right\}_{j<\omega}$ be a list of all the subsets of $G$ of cardinality $i$. Let $R$ be the following set of relations:

$$
\left\{c_{j}^{i} \cdot a_{i} \cdot y=y \cdot c_{j}^{i} \cdot a_{i} \mid i, j<\omega, y \in S_{j}^{i}\right\} .
$$

Then $\mathfrak{A}$ is obtained by starting with the free semigroup on the set $G$ of generators and then introducing the relations in $R$.

Since the only relations which we have added to the free semigroup on $G$ are of a "commuting" nature and in particular introduce no cancellation or reduction in the length of words, several properties follow. First, the indivisible members of $\mathfrak{A}$ are exactly the members of $G$; i.e., the formula $\sim\left(\exists x_{1}\right)\left(\exists x_{2}\right)\left(x_{1} \cdot x_{2}=x\right)$, denoted by $\psi(x)$, is satisfied in $\mathfrak{A}$ by, and only by, the members of $G$. Also, every member of $\mathfrak{A}$ is either indivisible or can be written as an indivisible times some other element; i.e.,

$$
\mathfrak{A} \vDash(\forall y)(\exists z)[\psi(z) \wedge(z=y \vee(\exists w)(y=z \cdot w))] .
$$

Let $\varphi_{n}(x)$ denote the formula

$$
\left(\forall y_{1}\right) \cdots\left(\forall y_{n}\right)(\exists z)\left[\psi(x) \wedge \psi(z) \wedge\left(\bigwedge_{i=1}^{n}\left(\psi\left(y_{i}\right) \rightarrow z \cdot x \cdot y_{i}=y_{i} \cdot z \cdot x\right)\right)\right] .
$$

The formula "says" of $x$ that it is indivisible and for any $y_{1}, \cdots, y_{n}$ there is an indivisible $z$ such that, for each $i$, if $y_{i}$ is indivisible then $z \cdot x \cdot y_{i}=y_{i} \cdot z \cdot x$. A was constructed in such a way that $\mathfrak{A} \vDash \varphi_{n}\left(a_{n}\right)$, the desired $z$ being any $c_{j}^{n}$ where $S_{j}^{n}$ contains all the indivisible $y_{i}$ 's. Furthermore, if $g_{1}, g_{2}, g_{3} \in G$ then $g_{1} \cdot g_{2} \cdot g_{3}=g_{3} \cdot g_{1} \cdot g_{2}$ in $\mathfrak{A}$ iff this relation is already in $R$ or $g_{1}=g_{2}=g_{3}$. We omit a proof of this; such a proof would first note that because there is no cancellation involved in the relations in $R$, words of length other then three could not be used to derive such a new relation, and then, 
by considering the various cases as to whether $g_{1}$ is some $a_{i}$ or some $c_{k}^{j}$ and so on, one could eliminate the different possibilities.

Hence for any $g \in G$ there is a positive integer $m$ and $g_{1}, \cdots, g_{m} \in G$ such that for any $g^{\prime} \in G$ there is at least one $i, 1 \leqq i \leqq m$, such that $g^{\prime} \cdot g \cdot g_{i} \neq g_{i} \cdot g^{\prime} \cdot g$. If $g=a_{i}$ then $m=i+1$ and $g_{1}, \cdots, g_{m}$ all different will suffice. But the set of formulas $T=\left\{\varphi_{n}(x)\right\}_{n<\omega}$ is finitely satisfiable in $\mathfrak{A}$ because, given any finite subset $T^{\prime}$ of $T$, if $n$ is the largest integer such that $\varphi_{n} \in T^{\prime}$ then clearly $a_{n}$ satisfies all the members of $T^{\prime}$.

By the Compactness Theorem for elementary first order logic there is a semigroup $\mathfrak{A}^{\prime}$ such that $\mathfrak{A}^{\prime}$ is denumerably infinite, $\mathfrak{A}^{\prime}>\mathfrak{A}$, and there is some $\bar{a} \in \mathfrak{Q}^{\prime}$ such that for each $n<\omega$, $\mathfrak{R}^{\prime} \vDash \varphi_{n}(\bar{a})$.

We will now show that $\mathfrak{B} * \mathfrak{A}$ and $\mathfrak{B} * \mathfrak{\mathfrak { Z } ^ { \prime }}$ are not elementarily equivalent and hence $\mathfrak{B} * \mathfrak{A}$ is not an elementary subsystem of $\mathfrak{B} * \mathfrak{\mathfrak { C } ^ { \prime }}$. Let $\theta$ be the sentence

$$
\begin{aligned}
& (\exists v)(\exists x)(\forall y)(\exists z)\left(\forall u_{1}\right)\left(\forall u_{2}\right)\left(\forall u_{3}\right)\{\psi(x) \wedge \psi(z) \wedge v \cdot v=v \\
\wedge & {\left[\left(y=u_{1} \cdot v \cdot u_{2} \cdot v \cdot u_{3} \wedge \sim\left(\exists u_{4}\right)\left(\exists u_{5}\right)\left(u_{2}=u_{4} \cdot v \cdot u_{5}\right)\right)\right.} \\
\rightarrow & \left.\left.(\exists w)(\exists r)\left(\left(u_{2}=w \vee u_{2}=w \cdot r\right) \wedge \psi(w) \wedge z \cdot x \cdot w=w \cdot z \cdot x\right)\right]\right\} .
\end{aligned}
$$

This sentence $\theta$, as it will be applied below, says roughly the following: There is an idempotent (which will have to be $b$ ) and an indivisible $x$ such that for any word $y$ we can find an indivisible $z$ such that for any way of writing $y$ as $u_{1} \cdot b \cdot u_{2} \cdot b \cdot u_{3}$ with $u_{2} \in A$ or $\in A^{\prime}$ (as the case may be), there is a left-most indivisible factor $w$ of $u_{2}$ such that $z \cdot x \cdot w=w \cdot z \cdot x$. We are using here the fact that $\mathfrak{B} * \mathfrak{R} \vDash \sim\left(\exists u_{4}\right)\left(\exists u_{5}\right)\left(u=u_{4} \cdot b \cdot u_{5}\right)$ iff $u \in A$, and similarly with $A^{\prime}$ in place of $A$.

We claim $\mathfrak{B} * \mathfrak{Q}^{\prime} \vDash \theta$ and $\mathfrak{B} * \mathfrak{Q} \vDash \sim \theta$. First, why $\mathfrak{B} * \mathfrak{Q}^{\prime} \vDash \theta$ ? Let $v$ be $b \in \mathfrak{B}$ and let $x$ be the $\bar{a} \in \mathfrak{X}^{\prime} . \quad \psi(\bar{a})$ holds in $\mathfrak{B} * \mathfrak{A}^{\prime}$ because $\bar{a}$ satisfies $\varphi_{1}(x)$ in $\mathfrak{Z}^{\prime}$. Now suppose $y \in \mathfrak{B} * \mathfrak{A}^{\prime}$ is given. We can assume $y$ is of the form $h_{1} \cdot b \cdot t_{1} \cdot b \cdot t_{2} \cdot b \cdot \cdots \cdot b \cdot t_{m} \cdot b \cdot h_{2}$ where $m \geqq 1$, each $t_{i} \in \mathfrak{X}^{\prime}$ and $h_{1}, h_{2}$ are each either $b$ or in $\mathfrak{X}^{\prime}$; otherwise the antecedent of $[\cdots \rightarrow \cdots]$ in $\theta$ could not be satisfied and we would easily finish. So for each $t_{i}$ there is an indivisible $w_{i} \in \mathfrak{A}^{\prime}$ such that either $t_{i}=w_{i}$ or $t_{i}=w_{i} \cdot r_{i}$ for some $r_{i} \in \mathfrak{A}^{\prime}$. (Recall every member of $\mathfrak{A}$ has this property and $\mathfrak{A} \equiv \mathfrak{U}^{\prime}$.) We know $\mathfrak{U}^{\prime} \vDash \varphi_{m}(\bar{a})$. So replacing $y_{i}$ in this formula by $w_{i}$ we get an indivisible $z \in \mathfrak{A}^{\prime}$ such that for all $i, 1 \leqq i \leqq m, z \cdot \bar{a} \cdot w_{i}=w_{i} \cdot z \cdot \bar{a}$. Now for any $u_{1}, u_{2}, u_{3}$ in $\mathfrak{B} * \mathfrak{A}^{\prime}$, if the antecedent of $[\cdots \rightarrow \cdots]$ is satisfied, it must be the case that for some $i, u_{2}=t_{i}$. So let $w$ be $w_{i}$, let $r$ be $r_{i}$ and we are done.

We now wish to show $\mathfrak{B} * \mathfrak{A} \vDash \sim \theta$. Suppose not. So we get $v$ and $x$. Since $v \cdot v=v$ we must have $v=b$ since $b$ is the only idempotent in $\mathfrak{B} * \mathfrak{A}$. Since $\mathfrak{B} * \mathfrak{A} \vDash \psi(x)$ we must have $x=g \in G$. 
As remarked above, there is an $m$ and $g_{1}, \cdots, g_{m} \in G$ such that for any $g^{\prime} \in G, \mathfrak{A} \vDash \sim \bigwedge_{i=1}^{m}\left(g^{\prime} \cdot g \cdot g_{i}=g_{i} \cdot g^{\prime} \cdot g\right)$. Let $y=b \cdot g_{1} \cdot b \cdot g_{2} \cdot \cdots \cdot b \cdot g_{m} \cdot b$. So we then get $z$. Since $\mathfrak{B} * \mathfrak{A} \vDash \psi(z)$, we must have $z \in G$; say $z=g^{\prime}$. Say $i_{0}$ is such that $g^{\prime} \cdot g \cdot g_{i_{0}} \neq g_{i_{0}} \cdot g^{\prime} \cdot g$. If $1<i_{0}<m$, let $u_{2}$ be $g_{i_{0}}$, let $u_{1}$ be $b \cdot g_{1} \cdot b \cdot \cdots \cdot b \cdot g_{i_{0}-1}$ and let $u_{3}$ be $g_{i_{0}+1} \cdot b \cdot \cdots \cdot b \cdot g_{m} \cdot b$. If $i_{0}=1$, let $u_{2}$ be $g_{i_{0}}$, let $u_{1}$ be $b$ and let $u_{3}$ be as above. The case $i_{0}=m$ is similar. Clearly the antecedent of $[\cdots \rightarrow \cdots]$ in $\theta$ is satisfied. So we get $w$ and $r$. It must be the case that $w=u_{2}\left(=g_{i_{0}}\right)$. But $z \cdot x \cdot w\left(=g^{\prime} \cdot g \cdot g_{i_{0}}\right) \neq w \cdot z \cdot x\left(=g_{i_{0}} \cdot g^{\prime} \cdot g\right)$, contradicting $\mathfrak{B} * \mathfrak{A} \vDash \theta$. So $\mathfrak{B} * \mathfrak{A} \vDash \sim \theta$.

We remark that in the logical hierarchy of formulas, $\theta$ is a $\Sigma_{6}$-sentence. It seems likely that an example showing that free products do not preserve elementary equivalence could be constructed in which the sentence $\theta$ is $\Sigma_{4}$, or perhaps $\Sigma_{3}$. It also seems likely that $\Sigma_{3}$ or at least $\Sigma_{2}$ equivalence is preserved by free products, and the method of games of the next result should suffice to show it.

A groupoid $\mathfrak{A}=\langle A, \cdot\rangle$ is a nonempty set $A$ and a function from $A \times A$ into $A$; and $*$ is now in the class of groupoids. We wish to show that if $\mathfrak{A}_{1}, \mathfrak{A}_{2}, \mathfrak{B}_{1}, \mathfrak{B}_{2}$ are groupoids, $\mathfrak{A}_{1} \equiv \mathfrak{B}_{1}, \mathfrak{A}_{2} \equiv \mathfrak{B}_{2}$, then $\mathfrak{H}_{1} * \mathfrak{A}_{2} \equiv \mathfrak{B}_{1} * \mathfrak{B}_{2}$. The method to be used is the method of games [1], [2], and the winning strategy for player II is similar to that which is used in showing that, as linearly ordered sets, $\omega \equiv \omega+\omega^{*}+\omega$. We wish to thank the referee whose questions and comments led to, among other things, improvement in the proof of the following theorem.

THEOREM. If $\mathfrak{A}_{1}, \mathfrak{A}_{2}, \mathfrak{B}_{1}, \mathfrak{B}_{2}$ are groupoids and $\mathfrak{A}_{1} \equiv \mathfrak{B}_{1}, \mathfrak{R}_{2} \equiv \mathfrak{B}_{2}$ then $\mathfrak{R}_{1} * \mathfrak{A}_{2} \equiv \mathfrak{B}_{1} * \mathfrak{B}_{2}$.

Proof. We can assume $A_{1} \cap A_{2}=B_{1} \cap B_{2}=\varnothing$. Let $n$ be a fixed positive integer. We will describe a winning strategy for player II in the game $G_{n}\left(\mathfrak{R}_{1} * \mathfrak{N}_{2}, \mathfrak{B}_{1} * \mathfrak{B}_{2}\right)$. We can assume that the nonlogical constants of the language are only $=$ (interpreted always as identity) and a three-place predicate $P$, where $P(a, b, c)$ means $a \cdot b=c$. This allows us to avoid considering terms. In the game, ${ }_{1} x,{ }_{2} x, \cdots,{ }_{n} x$ will be chosen from $\mathfrak{A}_{1} * \mathfrak{A}_{2}$ and ${ }_{1} y,{ }_{2} y, \cdots,{ }_{n} y$ from $\mathfrak{B}_{1} * \mathfrak{B}_{2}$. II wins iff for all $1 \leqq i, j, k \leqq n$

$$
{ }_{i} x \cdot{ }_{j} x={ }_{k} x \quad \text { iff } \quad{ }_{i} y \cdot{ }_{j} y={ }_{k} y
$$

and

$$
{ }_{i} x={ }_{j} x \quad \text { iff } \quad{ }_{i} y={ }_{j} y \text {. }
$$

We shall need II's winning strategy in $G_{m}\left(\mathfrak{A}_{1}, \mathfrak{B}_{1}\right)$ and in $G_{m}\left(\mathfrak{A}_{2}, \mathfrak{B}_{2}\right)$, where $m=\sum_{i=1}^{n} 2^{\left(3^{i}-2\right)}$. We require some notation. For each ${ }_{i} x$ in 
$\mathfrak{A}_{1} * \mathfrak{A}_{2}$, let ${ }_{i} x={ }_{i} x_{1}^{1}$. Then by induction, for $j \geqq 1$ and $k \geqq 1$, if ${ }_{i} x_{k}^{j} \in \mathfrak{A}_{1} * \mathfrak{A}_{2}-\left(\mathfrak{N}_{1} \cup \mathfrak{Q}_{2}\right)$, let ${ }_{i} x_{k}^{j}={ }_{i} x_{2 k-1}^{j+1} \cdot{ }_{i} x_{2 k}^{j+1}$. This decomposition is unique because of the definition of free product of groupoids. We say that each ${ }_{i} x_{k}^{j}$ is a factor of ${ }_{i} x_{k^{\prime}}^{j^{\prime}}$ if $j \geqq j^{\prime}$ and $2^{\left(j-j^{\prime}\right)}\left(k^{\prime}-1\right)+1 \leqq$ $k \leqq 2^{\left(j-j^{\prime}\right)}\left(k^{\prime}\right)$. And we say ${ }_{i} x_{k}^{j}$ has depth $j$ in ${ }_{i} x$. Similar notation is adopted for each ${ }_{i} y$. The $m$ defined above is the largest number of members of, say, $A_{1}$ which can appear among ${ }_{1} x, \cdots,{ }_{n} x$ at a depth $\leqq 3^{n}$ in ${ }_{1} x$ or $\leqq 3^{n-1}$ in ${ }_{2} x$ or $\cdots$ or $\leqq 3$ in ${ }_{n} x$. It is these members which are, in some sense, directly threatened by player I in the game. It will be convenient to assume first that $A_{1}, A_{2}, B_{1}, B_{2}$ are all infinite. We now begin to describe II's strategy.

First of all, if I chooses ${ }_{t} x$ then the ${ }_{t} y$ that II will choose will have exactly the same "form" as ${ }_{t} x$-i.e., ${ }_{t} y_{k}^{j} \in B_{1}$ iff ${ }_{t} x_{k}^{j} \in A_{1}$, and ${ }_{t} y_{k}^{j} \in B_{2}$ iff ${ }_{t} x_{k}^{j} \in A_{2}$. Similarly if I chooses ${ }_{t} y$.

Secondly, say I chooses ${ }_{1} x$ and ${ }_{1} x_{k_{1}}^{j_{1}}, \cdots,{ }_{1} x_{k_{p}}^{j_{p}}$ is a list of those factors of ${ }_{1} x$ which are at a depth $\leqq 3^{n}$ in ${ }_{1} x$ (i.e., each $j_{i} \leqq 3^{n}$ ) and which are in $A_{1}$. Then II chooses ${ }_{1} y_{k_{1}}^{j_{1}}, \cdots,{ }_{1} y_{k_{p}}^{j_{p}}$ from $B_{1}$ according to his winning strategy in $G_{m}\left(\mathfrak{N}_{1}, \mathfrak{B}_{1}\right)$. Note that $p \leqq 2^{\left(3^{n}-2\right)}$. A similar procedure is followed for such factors from $A_{2}$ and $B_{2}$. If I had chosen ${ }_{1} y$, again the procedure is similar. The method II uses for completing his choice of factors of ${ }_{1} y$ (if ${ }_{1} y$ is not already completely defined) will be specified later. It will not affect some parts of his later strategy, which it is convenient to give now. Say I chooses ${ }_{2} y$. Say ${ }_{2} y_{k_{1}}^{j_{1}}, \cdots,{ }_{2} y_{k_{r}}^{j_{r}}$ is a list of those factors of ${ }_{2} y$ which are in $B_{1}$ and which are at a depth $\leqq 3^{n-1}$ in ${ }_{2} y$. Note $r \leqq 2^{\left(3^{n-1}-2\right)}$. Then II chooses ${ }_{2} x_{k_{1}}^{j_{1}}, \cdots,{ }_{2} x_{k_{r}}^{j_{r}}$ from $A_{1}$ by using his winning strategy in $G_{m}\left(\mathfrak{A}_{1}, \mathfrak{B}_{1}\right)$ and taking into account the choices made in this game when ${ }_{1} x$ and ${ }_{1} y$ were discussed above. (So this involves choices number $p+1$ to $p+r$ in $G_{m}\left(\mathfrak{R}_{1}, \mathfrak{B}_{1}\right)$.) Similarly for factors of depth $\leqq 3^{n-1}$ in ${ }_{1} x$ and ${ }_{1} y$ which are from $A_{2}$ and $B_{2}$, and also similarly if $\mathrm{I}$ had chosen ${ }_{2} x$. Player II continues in this way for the rest of the game, choosing factors which are to be in $A_{1} \cup A_{2}$ or $B_{1} \cup B_{2}$ and at depth $\leqq 3^{n-d+1}$ in ${ }_{d} x$ according to his given winning strategies in the games $G_{m}$ and in the light of earlier choices in these games. Note that the choice of $m$ ensures that II has enough "room to work in". Note also that the winning strategy for II in these games $G_{m}\left(\mathfrak{A}_{1}, \mathfrak{B}_{1}\right)$ and $G_{m}\left(\mathfrak{A}_{2}, \mathfrak{B}_{2}\right)$ certainly includes maintaining equalities and inequalities - i.e., for those choices of factors specified above, we have ${ }_{t} x_{b}^{a}={ }_{r} x_{d}^{c}$ iff ${ }_{t} y_{b}^{a}={ }_{r} y_{d}^{c}$. This will be required later. Furthermore, at the end of the game, since the above winning strategies were used, we will have: If $i, j, k \leqq n$ then ${ }_{i} x,{ }_{j} x,{ }_{k} x$ are all in $A_{1}$ iff ${ }_{i} y,{ }_{j} y,{ }_{k} y$ are all in $B_{1}$, and in this case ${ }_{i} x={ }_{j} x$ iff ${ }_{i} y={ }_{j} y$ and and ${ }_{i} x \cdot{ }_{j} x={ }_{k} x$ iff ${ }_{i} y \cdot{ }_{j} y={ }_{k} y$. Similarly for $A_{2}$ and $B_{2}$.

We define for each $r, 0 \leqq r \leqq n$, conditions $K_{r}(1), \cdots, K_{r}(4)$ as follows: 
$K_{r}(1):$ Suppose $1 \leqq s<r$ and $j_{1}+j_{2}-1 \leqq 3^{n-r+1}$. Suppose ${ }_{r} x_{k_{1}}^{j_{1}}$ and ${ }_{8} x_{k_{2}}^{j_{2}}$ are defined and equal. Then ${ }_{r} y_{k_{1}}^{j_{1}}$ and ${ }_{s} y_{k_{2}}^{j_{2}}$ are also defined and equal.

$K_{r}(2)$ : Suppose $j_{1}+j_{2}-1 \leqq 3^{n-r+1}$ and suppose ${ }_{r} x_{k_{1}}^{j_{1}}$ and ${ }_{r} x_{k_{2}}^{j_{2}}$ are defined and equal. Then ${ }_{r} y_{k_{1}}^{j_{1}}$ and ${ }_{r} y_{k_{2}}^{j_{2}}$ are also defined and equal.

$K_{r}(3)$ : Replace "equal" in $K_{r}(1)$ by "unequal".

$K_{r}(4)$ : Replace "equal" in $K_{r}(2)$ by "unequal". Clearly $K_{0}(1), \cdots$, $K_{0}(4)$ are vacuously satisfied.

Assume that $1 \leqq t \leqq n$ and that ${ }_{1} x, \cdots,{ }_{t-1} x$ and ${ }_{1} y, \cdots,{ }_{t-1} y$ have been completely specified, following that part of II's strategy already indicated above and, for the rest (if any) of the factors in these elements, in such a way that for each $r, 0 \leqq r \leqq t-1, K_{r}(1), \cdots, K_{r}(4)$ are all satisfied. Suppose I chooses ${ }_{t} x$. Then for those factors of ${ }_{t} y$ which are to be in $B_{1} \cup B_{2}$ and at a depth $\leqq 3^{n-(t-1)}=3^{n-t+1}$ in ${ }_{t} y$, II specifies these factors according to that part of his strategy already given above. We now wish to show that II can complete his definition of ${ }_{t} y$ so that $K_{t}(1), \cdots, K_{t}(4)$ are all satisfied.

Consider those factors ${ }_{t} y_{k}^{j}$ of ${ }_{t} y$ which are in $B_{1} \cup B_{2}$ and with $j \leqq 3^{n-t+1}-$ i.e., those just specified by II. Conditions $K_{t}(1)$ and $K_{t}(3)$ might require some ${ }_{t} y_{b}^{a}$ equal or unequal to some ${ }_{r} y_{d}^{c}, r<t$ and $a+c-1 \leqq 3^{n-t+1}$; and this in turn might mean that there is a factor ${ }_{t} y_{k}^{j}$ of ${ }_{t} y_{b}^{a}$, which is a member of $B_{1} \cup B_{2}$, has $j \leqq 3^{n-t+1}$ (and thus was specified by II already) and which, if we are to have ${ }_{t} y_{b}^{a}$ equal or unequal to ${ }_{r} y_{d}^{c}$, will have to be equal or unequal (as the case may be) to ${ }_{r} y_{k_{1}}^{j_{1}}$, which is a factor of ${ }_{r} y_{d}^{c}$ and a member of $B_{1} \cup B_{2}$. Is this equality or inequality, needed for $K_{t}(1)$ or $K_{t}(3)$, satisfied? Assume it is equality we need. We have $a+c-1 \leqq 3^{n-t+1}, j \leqq 3^{n-t+1}$ and $c-j_{1}=a-j$. So $j_{1}=c+j-a \leqq c+j$. But $c \leqq c+a-1 \leqq 3^{n-t+1}$. So $j_{1} \leqq 3^{n-t+1}+3^{n-t+1} \leqq 3^{n-(t-1)+1} \leqq 3^{n-r+1}$. Hence ${ }_{r} x_{k_{1}}^{j_{1}}$ and ${ }_{r} y_{k_{1}}^{j_{1}}$ were "earlier moves" in the games $G_{m}$ being played and so, since ${ }_{t} x_{b}^{a}={ }_{r} x_{d}^{c}$ and thus ${ }_{t} x_{k}^{j}={ }_{r} x_{k_{1}}^{j_{1}}$, player II, as required by his winning strategies in the games $G_{m}$, chose ${ }_{t} y_{k}^{j}={ }_{r} y_{k_{1}}^{j_{1}}$. We have shown that the part of II's strategy already given does not conflict with conditions $K_{t}(1)$ and $K_{t}(3)$. The check that there is also no conflict with $K_{t}(2)$ and $K_{t}(4)$ is simpler and we do not give it.

We will now show how the rest (if any) of ${ }_{t} y$ is to be defined by II. Let ${ }_{t} x_{k_{1}}^{j_{1}}, \cdots,{ }_{t} x_{k_{p}}^{j_{p}}$ be a list of those factors of ${ }_{t} x$ which satisfy the hypothesis of condition $K_{t}(1)$ and such that no member of this list is a factor in ${ }_{t} x$ of any other (and hence there is no "overlap" among them at all, in the sense that they have no factors in common). It follows that every ${ }_{t} x_{k}^{j}$ which (together with some ${ }_{s} x_{k_{k}}^{j^{\prime}}, s<t$ ) satisfies the hypothesis of $K_{t}(1)$ is a factor of one of the members of this list.

Say ${ }_{t} x_{k_{1}}^{j_{1}}={ }_{r} x_{u}^{i}, r<t, j_{1}+i-1 \leqq 3^{n-t+1}$. For those ${ }_{t} x_{k^{\prime}}^{j^{\prime}}$ which are 
factors of ${ }_{t} x_{k_{1}}^{j_{1}}$, are in $A_{1} \cup A_{2}$ and satisfy $j^{\prime}>3^{n-t+1}$, II defines ${ }_{t} y_{k^{\prime}}^{j^{\prime}}$, so that ${ }_{t} y_{k_{1}}^{j_{1}}={ }_{r} y_{u}^{i}$. There is no difficulty in doing this. However, suppose ${ }_{t} x_{k}^{j}$ is a factor of ${ }_{t} x_{k_{1}}^{j_{1}}$ and suppose ${ }_{t} x_{k}^{j}$ and ${ }_{s} x_{b}^{a}(s<t)$ satisfy the hypothesis of $K_{t}(1)$. Since ${ }_{t} y_{k}^{j}$ has just been defined (since it is a piece of $\left.{ }_{t} y_{k_{1}}^{j_{1}}\right)$, does it satisfy the conclusion of $K_{t}(1)$ ? We have $j+$ $a-1 \leqq 3^{n-t+1}$. We require ${ }_{t} y_{k}^{j}={ }_{s} y_{b}^{a}$. Since ${ }_{t} x x_{k_{1}}^{j_{1}}={ }_{r} x_{u}^{i}$ and since they have exactly the same form, let ${ }_{t} x x_{u^{\prime}}^{i^{\prime}}$ be that factor of ${ }_{r} x_{u}^{i}$ which corresponds to ${ }_{t} x_{k}^{j}$ under the correspondence given by this "sameness of form". Of course ${ }_{r} x_{u^{\prime}}^{i^{\prime}}={ }_{t} x_{k}^{j}$ and $i^{\prime}-i=j-j_{1}$. In the same way, ${ }_{t} y_{k}^{j}={ }_{r} y_{u^{\prime}}^{i^{\prime}} . \quad$ But $a+j_{1}+\left(j-j_{1}\right)-1=a+j-1 \leqq 3^{n-t+1}$ and this implies $a+\left(j-j_{1}\right) \leqq 3^{n-t+1}$. Also $j_{1}+i-1 \leqq 3^{n-t+1}$ implies $i \leqq 3^{n-t+1}$. Hence $a+i^{\prime}=a+\left(i^{\prime}-i\right)+i=a+\left(j-j_{1}\right)+i \leqq 2 \cdot 3^{n-t+1} \leqq 3^{n-(t-1)+1} \leqq$ $3^{n-\max (s, r)+1}$. So by the induction hypothesis for $K_{\max (s, r)}(1)$ if $r \neq s$ or for $K_{s}(2)$ if $r=s$, we get ${ }_{s} y_{b}^{a}={ }_{r} y_{u^{\prime}}^{i^{\prime}}$. So ${ }_{s} y_{b}^{a}={ }_{t} y_{k}^{j}$.

Suppose ${ }_{t} x_{k_{1}}^{j_{1}}={ }_{r} x_{u}^{i}$ (as above) and in addition ${ }_{t} x_{k_{1}}^{j_{1}}={ }_{v} x_{d}^{c}$ with $v<t$ and $j_{1}+c-1 \leqq 3^{n-t+1}$. Then by an argument similar to the above, we could show ${ }_{v} y_{d}^{c}={ }_{r} y_{u}^{i}$ and so no conflict arises here.

Player II now repeats the above procedure for ${ }_{t} x_{k_{1}}^{j_{1}}$ on ${ }_{t} x_{k_{2}}^{j_{2}}, \cdots,{ }_{t} x_{k p}^{j p}$. Since, as remarked above, no two of these overlap there is no difficulty in making the definitions to satisfy $K_{t}(1)$; and again as above, factors of members of this list are automatically taken care of.

We now wish to consider condition $K_{t}(2)$. Suppose ${ }_{t} x_{k_{1}}^{j_{1}}$ and ${ }_{t} x x_{k_{2}}^{j_{2}}$ satisfy the hypothesis of $K_{t}(2)$ (i.e., they are equal and $j_{1}+j_{2}-1 \leqq 3^{n-t+1}$ ). Suppose further that there is no pair of factors of ${ }_{t} x$ satisfying the hypothesis of $K_{t}(2)$ and with either of these factors being factors of ${ }_{t} x_{k_{1}}^{j_{1}}$ or of ${ }_{t} x_{k_{2}}^{j_{2}}$-i.e., this latter pair is "minimal" with respect to the hypothesis of $K_{t}(2)$. If there are any pairs satisfying the hypothesis of $K_{t}(2)$ then there is a minimal pair because: If ${ }_{t} x_{k_{1}}^{j_{1}}{ }_{t} x_{k_{2}}^{j_{2}}$ and ${ }_{t} x_{k_{3}}^{j_{3}},{ }_{t} x_{k_{4}}^{j_{4}}$ are different pairs satisfying the hypothesis of $K_{t}(2)$ and if ${ }_{t} x_{k_{1}}^{j_{1}}$ is a factor of ${ }_{t} x_{k_{3}}^{j_{3}}$ then if ${ }_{t} x_{k_{4}}^{j_{4}}$ were a factor of ${ }_{t} x_{k_{2}}^{j_{2}}$, we would get ${ }_{t} x_{k_{2}}^{j_{2}}$ equal to a proper factor of itself and this is impossible in a free product. So we can "work our way down in depth" and consider a minimal pair ${ }_{t} x_{k_{1}}^{j_{1}},{ }_{t} x_{k_{2}}^{j_{2}}$. Player II must arrange ${ }_{t} y_{k_{1}}^{j_{1}}={ }_{t} y_{k_{2}}^{j_{2}}$. There are several ways in which the parts of ${ }_{t} y$ defined in satisfying $K_{t}(1)$ might conflict with this desired result.

Suppose ${ }_{t} y_{k^{\prime}}^{j^{\prime}}={ }_{r} y_{u^{\prime}}^{i^{\prime}}, i^{\prime}+j^{\prime}-1 \leqq 3^{n-t+1}, r<t$, and ${ }_{t} y_{k^{\prime \prime}}^{j^{\prime \prime}}={ }_{s} y_{u^{\prime \prime}}^{i^{\prime \prime}} j^{j^{\prime \prime}}+$ $i^{\prime \prime}-1 \leqq 3^{n-t+1}, s<t$ were arranged in satisfying $K_{t}(1)$. Say ${ }_{t} y_{k_{1}}^{j_{1}}$ is a factor of ${ }_{t} y_{k^{\prime}}^{j^{\prime}}$ and ${ }_{t} y_{k_{2}}^{j_{2}}$ is a factor of ${ }_{t} y_{k^{\prime \prime}}^{j^{\prime \prime}}$; hence ${ }_{t} y_{k_{1}}^{j_{1}}$ and ${ }_{t} y_{k_{2}}^{j_{2}}$ have already been completely defined. Let ${ }_{r} y_{b}^{a}$ be the factor of ${ }_{r} y_{u^{\prime}}^{j^{\prime}}$ which corresponds (under the sameness-of-form correspondence between ${ }_{r} y_{u^{\prime}}^{i^{\prime}}$ and $\left.{ }_{r} y_{k^{\prime}}^{j^{\prime}}\right)$ to ${ }_{t} y_{k_{1}}^{j_{1}}$. Similarly for ${ }_{s} y_{d}^{c}$ and ${ }_{t} y_{k_{2}}^{j_{2}}$. It suffices to show ${ }_{r} y_{b}^{a}={ }_{s} y_{d}^{c}$. We have $i^{\prime}-a=j^{\prime}-j_{1}, i^{\prime \prime}-c=j^{\prime \prime}-j_{2}, i^{\prime}+j^{\prime}-1 \leqq$ $3^{n-t+1}, i^{\prime \prime}+j^{\prime \prime}-1 \leqq 3^{n-t+1}$, and $j_{1}+j_{2}-1 \leqq 3^{n-t+1}$. So $i^{\prime} \leqq 3^{n-t+1}, i^{\prime \prime} \leqq$ $3^{n-t+1}$ and $\left(j_{1}-j^{\prime}\right)+j^{\prime}+\left(j_{2}-j^{\prime \prime}\right)-j^{\prime \prime}-1 \leqq 3^{n-t+1}$. Hence $\left(j_{1}-j^{\prime}\right)+$ 
$\left(j_{2}-j^{\prime \prime}\right)-1 \leqq 3^{n-t+1}$. But then $a+c-1=i^{\prime}+j_{1}-j^{\prime}+i^{\prime \prime}+j_{2}-$ $j^{\prime \prime}-1 \leqq 3 \cdot 3^{n-t+1}=3^{n-(t-1)+1} \leqq 3^{n-\max (r, s)+1}$. So by the induction hypothesis, using $K_{\max (r, s)}(1)$ if $s \neq r$ and $K_{s}(2)$ if $s=r$, we get ${ }_{r} y_{b}^{a}={ }_{s} y_{d}^{c}$.

Now suppose things are as above, except ${ }_{t} y_{k^{\prime \prime}}^{j^{\prime \prime}}$ is a factor of ${ }_{t} y_{k_{2}}^{j_{2}}$ instead of the other way round. By computations very similar to those above we would get ${ }_{t} y_{k^{\prime \prime}}$, equal to its corresponding (under the correspondence determined by ${ }_{t} x_{k_{1}}^{j_{1}}$ being equal to $\left.{ }_{t} x_{k_{2}}^{j_{2}}\right)$ image in ${ }_{t} y_{k_{1}}^{j_{1}}$. Player II then defines those ${ }_{t} y_{b}^{a}$ which are factors of ${ }_{t} y_{k_{2}}^{j_{2}^{2}}$, which are required to be in $B_{1} \cup B_{2}$, and which are not already defined, by making them equal to the corresponding (same correspondence) factors in ${ }_{t} y_{k_{1}}^{j_{1}}$, all of which were defined earlier.

Now suppose things are as above, except ${ }_{t} y_{k^{\prime}}^{j^{\prime}}$ is a factor of ${ }_{t} y_{k_{1}}^{j_{1}}$ and ${ }_{t} y_{k^{\prime \prime}}^{j^{\prime \prime}}$ is a factor of ${ }_{t} y_{k_{2}}^{j_{2}}$. There are now several subcases to consider, depending on how these factors overlap under the correspondence determined by ${ }_{t} x_{k_{1}}^{j_{1}}$ being equal to ${ }_{t} x_{k_{2}}^{j_{2}}$. All of them involve computations similar to the one given above, and we omit them. Once the definitions made in satisfying $K_{t}(1)$ are seen not to conflict with ${ }_{t} y_{k_{1}}^{j_{1}}$ being equal to ${ }_{t} y_{k_{2}}^{j_{2}}$, II can define those factors of, say, ${ }_{t} y_{k_{1}}^{j_{1}}$ which are to be in $B_{1} \cup B_{2}$ and which are supposed to equal factors already defined in ${ }_{t} y_{k_{2}}^{j_{2}}$. One possibility remains. A certain factor of ${ }_{t} y_{k_{1}}^{j_{1}}$ is to be a member of $B_{1} \cup B_{2}$ and equal to its corresponding factor in ${ }_{t} y_{k_{2}}^{j_{2}}$ - but neither has been defined by any of the above considerations. In this case we use the assumption that $B_{1}$ and $B_{2}$ are each infinite, and II chooses any member (from $B_{1}$ or $B_{2}$, whichever is needed so that ${ }_{t} y$ and ${ }_{t} x$ will have the same form) which is completely new - i.e., which appears nowhere in ${ }_{1} x, \cdots,{ }_{t} x,{ }_{1} y, \cdots,{ }_{t-1} y$ and that part of ${ }_{t} y$ so far defined. This completes the definition of ${ }_{t} y_{k_{1}}^{j_{1}}$ and ${ }_{t} y_{k_{2}}^{j_{2}}$.

Other minimal pairs satisfying the hypothesis of $K_{t}(2)$ are handled similarly. We then consider pairs which are minimal in the sense above, but with respect only to those pairs not yet considered. The arguments are analogous, and II proceeds to define as much of ${ }_{t} y$ as is required to satisfy $K_{t}(2)$.

We have thus defined part of ${ }_{t} y$ and at the same time shown that $K_{t}(1)$ and $K_{t}(2)$ are satisfied. For the remaining (if any) factors of ${ }_{t} y$ which are to be members of $B_{1} \cup B_{2}$ we again use the assumption that $B_{1}$ and $B_{2}$ are infinite and II chooses completely new elements. It follows that $K_{t}(3)$ and $K_{t}(4)$ are thus satisfied.

If player I had picked ${ }_{t} y$ then $K_{t}(1), \cdots, K_{t}(4)$ and II's strategy are gotten by interchanging $x$ and $y$.

Now suppose not all of $A_{1}, A_{2}, B_{1}, B_{2}$ are infinite. If $A_{1}$ is finite, since $\mathfrak{N}_{1} \equiv \mathfrak{B}_{1}$, we get $f: \mathfrak{Q}_{1} \cong \mathfrak{B}_{1}$. If in addition $A_{2}$ is finite, we get $\mathfrak{A}_{1} * \mathfrak{A}_{2} \cong \mathfrak{B}_{1} * \mathfrak{B}_{2}$. So assume $A_{2}$ (and hence also $B_{2}$ ) is infinite. Then player II's winning strategy is modified so that if I chooses ${ }_{t} x$ and 
${ }_{t} x_{k}^{j} \in A_{1}$ then II defines ${ }_{t} y_{k}^{j}=f\left({ }_{t} x_{k}^{j}\right)$, and similarly if I chooses ${ }_{t} y$. It can be shown that all of the conclusions above are obtained also in this situation.

Now suppose the game is over and ${ }_{1} x, \cdots,{ }_{n} x,{ }_{1} y, \cdots,{ }_{n} y$ have been chosen. We want to show, for $1 \leqq i, j, k \leqq n$, that ${ }_{i} x \cdot{ }_{j} x={ }_{k} x$ iff ${ }_{i} y \cdot{ }_{j} y={ }_{k} y$ and ${ }_{i} x={ }_{j} x$ iff ${ }_{i} y={ }_{j} y$. As noted above, if ${ }_{i} x,{ }_{j} x,{ }_{k} x$ are all in $A_{1}$ then ${ }_{i} y,{ }_{j} y,{ }_{k} y$ are all in $B_{1}$, and conversely, and the result then follows from II's winning strategy in $G_{m}\left(\mathfrak{A}_{1}, \mathfrak{B}_{1}\right)$. Similarly for $A_{2}$ and $B_{2}$. So now assume this is not the case. There are now several cases to consider; we discuss two of them.

(i) Suppose $i<k<j$ and ${ }_{i} x \cdot{ }_{j} x={ }_{k} x$. So ${ }_{k} x_{1}^{2}={ }_{i} x_{1}^{1}$ and ${ }_{k} x_{2}^{2}={ }_{j} x x_{1}^{1}$. Since $j \leqq n$ and $k \leqq n$ we have $3^{n-k+1} \geqq 2$ and $3^{n-j+1} \geqq 2$. And so $2+1-1 \leqq 3^{n-k+1}$ and $2+1-1 \leqq 3^{n-j+1}$. Thus conditions $K_{k}(1)$, $K_{k}(3), K_{j}(1), K_{j}(3)$ ensure that ${ }_{k} y_{1}^{2}={ }_{i} y_{1}^{1}$ and ${ }_{k} y_{2}^{2}={ }_{j} y_{1}^{1}$, and thus ${ }_{k} y=$ ${ }_{i} y \cdot{ }_{j} y$.

(ii) Suppose $i>k$ and ${ }_{i} x \cdot{ }_{i} x={ }_{k} x$. So ${ }_{k} x_{1}^{2}={ }_{k} x_{2}^{2}={ }_{i} x$. Again $k \leqq n$, so $3 \leqq 3^{n-k+1}$, so $2+2-1 \leqq 3^{n-k+1}$. Thus condition $K_{k}(2)$ and $K_{k}(4)$ ensured that, when ${ }_{k} x$ and ${ }_{k} y$ were chosen, we had ${ }_{k} x_{1}^{2}={ }_{k} x_{2}^{2}$ iff ${ }_{k} y_{1}^{2}={ }_{k} y_{2}^{2}$. Then, as in case (i) above, conditions $K_{i}(1)$ and $K_{i}(3)$ ensure ${ }_{i} x={ }_{k} x_{1}^{2}={ }_{k} x_{2}^{2}$ iff ${ }_{i} y_{1}^{2}={ }_{k} y_{2}^{2}={ }_{i} y$.

The other cases are no more difficult.

REMARK. For any positive integer $n$, let $m$ be defined (as a function of $n$ ) as in the proof of the above theorem. Let $\equiv_{p}$ mean equivalence with respect to sentences with at most $p$ variables. Then in fact the above proof shows that:

(1) if $\mathfrak{U}_{1}, \mathfrak{U}_{2}, \mathfrak{B}_{1}, \mathfrak{B}_{2}$, are all infinite,

$$
\mathfrak{Q}_{1} \equiv{ }_{m} \mathfrak{B}_{1}, \mathfrak{Q}_{2} \equiv_{m} \mathfrak{B}_{2} \text {, then } \mathfrak{U}_{1} * \mathfrak{Q}_{2} \equiv_{n} \mathfrak{B}_{1} * \mathfrak{B}_{2} \text {. }
$$

(2) if $\mathfrak{U}_{1} \cong \mathfrak{B}_{1}, \mathfrak{R}_{2}$ and $\mathfrak{B}_{2}$ are infinite, and

$$
\mathfrak{R}_{2} \equiv{ }_{m} \mathfrak{S}_{2} \text { then } \mathfrak{R}_{1} * \mathfrak{N}_{2} \equiv{ }_{n} \mathfrak{B}_{1} * \mathfrak{B}_{2} \text {. }
$$

It seems likely that these last results could be strengthened - in particular by weakening the hypotheses.

CoROLlaRY. If $\mathfrak{A}_{1}, \mathfrak{A}_{2}, \mathfrak{B}_{1}, \mathfrak{B}_{2}$ are groupoids, $\mathfrak{R}_{2} \prec \mathfrak{B}_{2}, \mathfrak{N}_{1} \prec \mathfrak{B}_{1}$, then $\mathfrak{A}_{1} * \mathfrak{H}_{2} \prec \mathfrak{B}_{1} * \mathfrak{B}_{2}$.

The proof of the corollary is essentially the same as that for the theorem, except that we start with ${ }_{1} x={ }_{1} y, \cdots,{ }_{p} x={ }_{p} y$ for some fixed $p<n$, and the first part of II's strategy is modified to use II's winning strategy gotten from the games appropriate for $\mathfrak{H}_{1} \prec \mathfrak{B}_{1}$ and $\mathfrak{A}_{2} \prec \mathfrak{B}_{2}$.

What follows is a short attempt to motivate intuitively the 
above results. The major reason the preservation result is true for groupoids is that, because of the lack of an associative law, factors of an $x \in \mathfrak{A} * \mathfrak{B}-(\mathfrak{A} \cup \mathfrak{B})$ which are inside a sufficient number of brackets cannot be "connected" with $x$ in a game with only $n$ rounds. For example, to "state" that ${ }_{1} x_{k}^{j}$ is a factor of ${ }_{1} x$, player I would need at least $j$ rounds. If $G_{n}$ is being played and $n<j$, player II knows that I cannot do it. So when ${ }_{1} x$, in the game $G_{n}$, has been chosen, only members of the original groupoids at a depth $\leqq 3^{n}$ in ${ }_{1} x$ are "threatened" by I; for the others it suffices that II maintain certain equalities and inequalities.

However, for semigroups an element, say $a_{1} \cdot b_{1} \cdot a_{2} \cdot b_{2} \cdots \cdots a_{n} \cdot b_{n}$ in $\mathfrak{A} * \mathfrak{B}$, does not depend on the bracketing. And the $b_{i}$ 's are all equally and quickly "accessible". Thus in round 1, player II commits himself to some choice and in round 2 player I can then present II with an arbitrarily large finite subset of elements, any one of which is accessible in 2 or 3 more rounds. The above counterexample for semigroups takes advantage of this, as well as the idea in Example 1.3 of [5].

\section{REFERENCES}

1. A. Ehrenfeucht, An application of games to the completeness problem for formalized thories, Fund. Math., 49 (1961), 129-141.

2. S. Feferman, Infinitary Properties, Local Functors, and Systems of Ordinal Functions, Conference in Mathematical Logic - London 1970, Lecture Notes in Mathematics, no. 255, Springer-Verlag, 1972, 63-97.

3. S. Feferman and R. L. Vaught, The first order properties of products of algebraic systems, Fund. Math., 47 (1959), 57-103.

4. P. Olin, Direct multiples and powers of modules, Fund. Math., 73 (1971), 113-124.

5. - Products of two-sorted structures, J. Symbolic Logic, 37 (1972), 75-80.

6. - Free products and elementary equivalence, Notices of Amer. Math. Soc., 20 (1973), A-284.

Received January 15, 1973. This work was partially supported by the National Research Council of Canada.

York University, TORONTO, CANADA 


\section{PACIFIC JOURNAL OF MATHEMATICS}

EDITORS

RICHARD ARENS (Managing Editor)

University of California

Los Angeles, California 90024

R. A. Beaumont

University of Washington

Seattle, Washington 98105

J. DugundJI

Department of Mathematics

University of Southern California

Los Angeles, California 90007

D. Gilbarg and J. Milgram

Stanford University

Stanford, California 94305

\section{ASSOCIATE EDITORS}

E. F. BECKENBACH

B. H. NeumanN

F. WOLF

K. YOSHIDA

\section{SUPPORTING INSTITUTIONS}

UNIVERSITY OF BRITISH COLUMBIA CALIFORNIA INSTITUTE OF TECHNOLOGY

UNIVERSITY OF CALIFORNIA

MONTANA STATE UNIVERSITY

UNIVERSITY OF NEVADA

NEW MEXICO STATE UNIVERSITY

OREGON STATE UNIVERSITY

UNIVERSITY OF OREGON

OSAKA UNIVERSITY

\author{
UNIVERSITY OF SOUTHERN CALIFORNIA \\ STANFORD UNIVERSITY \\ UNIVERSITY OF TOKYO \\ UNIVERSITY OF UTAH \\ WASHINGTON STATE UNIVERSITY \\ UNIVERSITY OF WASHINGTON \\ $\stackrel{*}{*} \stackrel{*}{*}{ }^{*}{ }^{*}{ }^{2}$ AMERICAN MATHEMATICAL SOCIETY \\ NAVAL WEAPONS CENTER
}




\section{Pacific Journal of Mathematics}

\section{Vol. 52, No. $1 \quad$ January, 1974}

David R. Adams, On the exceptional sets for spaces of potentials ............ 1

Philip Bacon, Axioms for the Čech cohomology of paracompacta ............ 7

Selwyn Ross Caradus, Perturbation theory for generalized Fredholm operators ..... 11

Kuang-Ho Chen, Phragmén-Lindelöf type theorems for a system of nonhomogeneous equations ............................ 17

Frederick Knowles Dashiell, Jr., Isomorphism problems for the Baire classes .......

M. G. Deshpande and V. K. Deshpande, Rings whose proper homomorphic images are right subdirectly irreducible . . . . . . . . . . . . . . . . . . . . . . . . .

Mary Rodriguez Embry, Self adjoint strictly cyclic operator algebras .............

Paul Erdős, On the distribution of numbers of the form $\sigma(n) / n$ and on some related

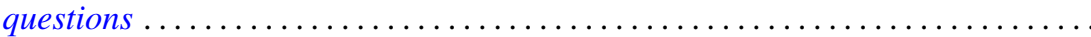

Richard Joseph Fleming and James E. Jamison, Hermitian and adjoint abelian

operators on certain Banach spaces ............................

Stanley P. Gudder and L. Haskins, The center of a poset .................. 85

Richard Howard Herman, Automorphism groups of operator algebras . . . ........

Worthen N. Hunsacker and Somashekhar Amrith Naimpally, Local compactness of families of continuous point-compact relations ....................

Donald Gordon James, On the normal subgroups of integral orthogonal groups ....

Eugene Carlyle Johnsen and Thomas Frederick Storer, Combinatorial structures in

loops. II. Commutative inverse property cyclic neofields of prime-power

order.

Ka-Sing Lau, Extreme operators on Choquet simplexes . . . . . . . . . . . . . . 129

Philip A. Leonard and Kenneth S. Williams, The septic character of 2, 3, 5 and $7 \ldots 143$

Dennis McGavran and Jingyal Pak, On the Nielsen number of a fiber map ........ 149

Stuart Edward Mills, Normed Köthe spaces as intermediate spaces of $L_{1}$ and

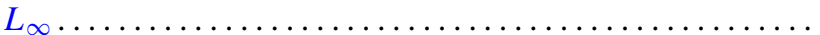

Philip Olin, Free products and elementary equivalence. .

Louis Jackson Ratliff, Jr., Locally quasi-unmixed Noetherian rings and ideals of the principal class.

Seiya Sasao, Homotopy types of spherical fibre spaces over spheres ...

Helga Schirmer, Fixed point sets of polyhedra ...

Kevin James Sharpe, Compatible topologies and continuous irreducible

representations.

Frank Siwiec, On defining a space by a weak base . . . . . . . . . . . . . . . 233

James McLean Sloss, Global reflection for a class of simple closed curves ....... 247

M. V. Subba Rao, On two congruences for primality . .

Raymond D. Terry, Oscillatory properties of a delay differential equation of even

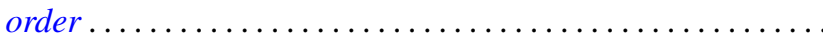

Joseph Dinneen Ward, Chebyshev centers in spaces of continuous functions . .

Robert Breckenridge Warfield, Jr., The uniqueness of elongations of Abelian

groups...

V. M. Warfield, Existence and adjoint theorems for linear stochastic differential

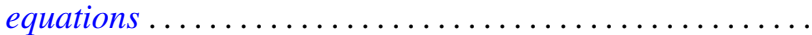

